\title{
Steady distribution of cells in a one-dimensional biased random walk model of bacterial chemotaxis
}

\author{
Tomonobu GOTO* and Tonau NAKAI * \\ *Department of Mechanical and Aerospace Engineering, Tottori University \\ 4-101 Koyama-Minami, Tottori 680-8552, Japan \\ E-mail:goto@damp.tottori-u.ac.jp
}

Received 23 October 2015

\begin{abstract}
Most bacteria are motile, moving toward suitable environments for subsistence and reproduction. In isotropic chemical environments, they move randomly, changing direction at regular time intervals. In the presence of chemical gradients, they modulate the frequency of the direction change. Collectively, this modulation constitutes the chemotactic response toward a desirable chemical. This study investigates a one-dimensional discrete biased random walk model based on bacterial chemotaxis; a modified version of the classical random walk model. Each cell in the group moves along a uniformly spaced number line at the rate of one interval per time step. A chemical attractant is placed at the origin of the number line. When a cell has receded from the origin in the previous time step, it changes its direction with a probability of $1 / 2$ in the current time step. On the other hand, when a cell has approached the origin in the previous time step, its direction changes with probability $(1-\alpha) / 2$, where $\alpha$ denotes the intensity of the bias toward the origin. In numerical simulations, the cells establish a steady distribution from the origin. This distribution is expressed using a geometric progression whose common ratio depends on $\alpha$. We provide an analytical explanation of this distribution, which actually constitutes two steady distributions alternating at odd and even positions. Next, the results of the discrete model are compared with those of the corresponding continuum model, namely, a diffusion-advection equation wherein $\alpha$ determines the advection speed. The theoretical solution of the diffusion-advection equation is an exponential decay function, consistent with the distribution obtained by the discrete model.
\end{abstract}

Key words : Biased random walk, Discrete model, Bacterial chemotaxis, Collective behavior, Steady distribution, Geometric progression, Diffusion advection equation

\section{Introduction}

Bacterial chemotaxis occurs via a sequence of runs and tumbles (e.g., Adler, 1966, Rubik and Koshland, 1978). A "run" refers to a smooth swimming motion, and a "tumble" indicates a directional change. In aqueous media, run and tumble motions are randomized. However, near a chemical attractant, a cell reduces the frequency of its tumbles (Macnab and Koshland, 1972), which biases its motion toward the source.

A bacterial cell is too small to sense the spatial distribution of the chemical attractant at a given time using two or more sensors (Berg, 2004). Thus, bacterial chemotactic behavior results from three distinct mechanisms.

First, by moving a certain distance, the bacterium can detect whether it is approaching or receding from the chemoattractant source. The cell senses and compares the chemical concentration at two consecutive times. If the concentration has increased or decreased at the later time, the cell has approached or receded from the source, respectively. Second, the cell randomly determines its direction after a tumble. The direction is changed by fluid dynamic interactions between the deforming flagella and the cell body. Although the process of tumbling is well understood (Turner, et al., 2000), the prediction of tumbling remains a complex fluid dynamics problem (Kanehl and 
Ishikawa, 2014). Therefore, a bacterial cell cannot choose a proper posture relative to the source. Third, the cell does not stop when it detects the maximal concentration. Because the cell might be smaller than the spatial variability of the concentration distribution, continued motion is a suitable strategy to avoid trapping in local concentration maxima.

In the present study, we introduce a parameter $\alpha$, which denotes the intensity of the bias, into a previously proposed one-dimensional discrete model (Goto and Nakai, 2015). A cell moves along a number line, as in the classical random walk model (e.g., Berg, 1993). The model applies two very simple rules based on real chemotactic behavior: if a cell recedes from the origin in one time step, it randomly moves right or left with a probability of $1 / 2$, and if a cell approaches the origin in one time step, it either moves in the same direction in the next time step with probability $\alpha$ or randomly selects its direction with probability $1-\alpha$. When many cells initially reside at the origin, their biased random motions following these rules eventually lead to a steady distribution.

The model is a slight modification of the recurrence relations related to the telegraph equation. To include the tendency of motion toward one preferable direction, recurrence relations between the fraction of cells at a position at the present time and the fraction of cells at the neighboring positions at the previous time were introduced into the classical random walk model. (Goldstein, 1951, Kac, 1974). These were used to derive a continuum model leading to the telegraph equation. In the present model, the recurrence relations will be slightly modified considering the difference in the direction change probabilities of right-moving and left-moving cells. The difference was considered also when the biased telegraph equation was derived from the discrete model (Schnitzer, 1993, Codling, et al., 2008), although the recurrence relations do not explicitly appear. In the present study, a diffusion-advection equation will be derived as a continuum model.

Section 2 develops the model and shows how the modified rules lead to a steady distribution of cells around the origin. The dependence of this distribution on $\alpha$ is analytically explained. Section 3 compares the solutions of the modified discrete model and the corresponding continuum model. Section 4 discusses the difference between the steady distribution obtained by the biased telegraph equation and the steady distribution by the present model.

\section{Steady probability distributions}

\subsection{One-dimensional biased random walk model}

Let us assume that a cell moves along a number line sectioned at uniform intervals $a$ at a rate of one interval per time step, as described in the previous model, as is shown in Fig. 1. The chemical attractant is sourced from the origin.

The rules of cell motion are based on the three mechanisms of bacterial chemotaxis mentioned in Section 1. First, as the cell moves, it senses whether it approaches or recedes from the origin. Second, when the cell senses movement away from the origin, it randomly changes direction. Third, the cell does not stop at the origin. These behaviors are

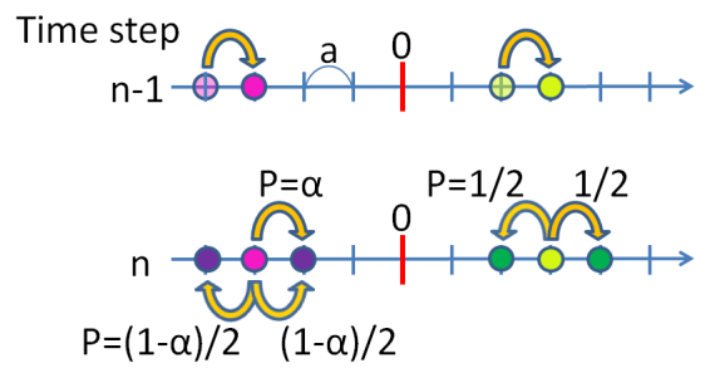

Fig. 1. Biased random walk model. In each time step, the cell moves along a number line by one interval to the left or right. When a cell has receded from the origin at time $n-1$ (green), it selects its direction with probability $1 / 2$. When the cell has approached to the origin at time $n-1$ (purple), the probability that the cell changes direction reduces from 1 to $1-\alpha$. The cell continues toward the origin with probability $\alpha$. 
embodied in two biased rules: (a) If a cell has receded from the origin in the previous time step, it moves to the left or right with the same probability $(1 / 2)$. (b) If the cell has approached the origin in the previous time step, it either continues in the same direction with probability $\alpha$ or randomly selects a new direction with probability $1-\alpha$. The total probability that a cell will maintain or reverse its direction is $1 / 2 \cdot(1+\alpha)$ and $1 / 2 \cdot(1-\alpha)$, respectively. When $\alpha=1$, the model reduces to the previous biased random walk model (Goto and Nakai, 2015), and when $\alpha=0$, it reduces to the classical random walk.

Rule (a) corresponds to a tumbling motion, wherein the cell randomly changes its swimming direction. Rule (b) corresponds to the reduction in tumbling frequency when the cell senses a higher concentration of attractant chemical. If many cells move stochastically under the two biased rules, they will eventually gather around the origin. This accumulation corresponds to the spatial distribution of bacterial cells produced by the chemotaxis.

(a)

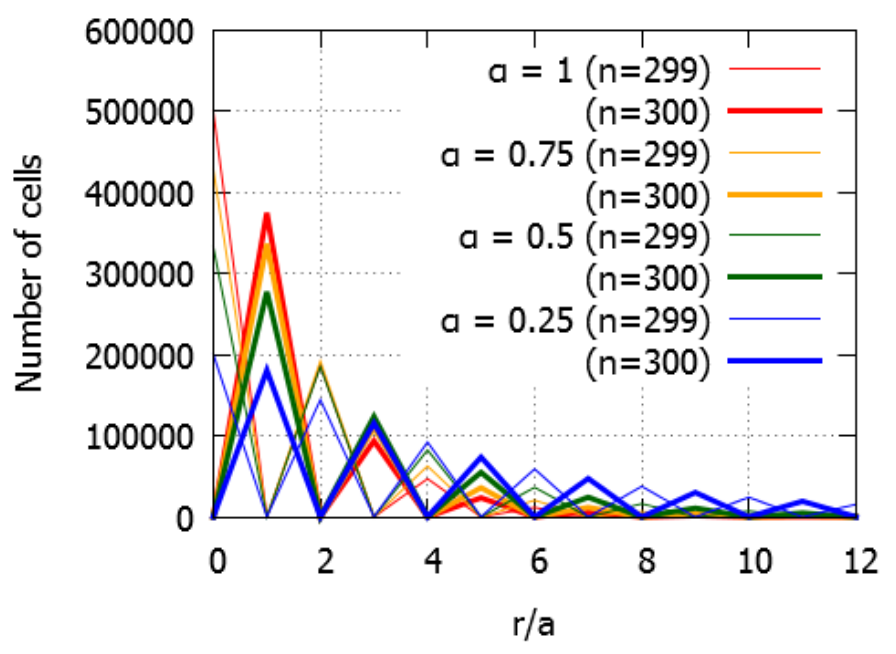

(b)

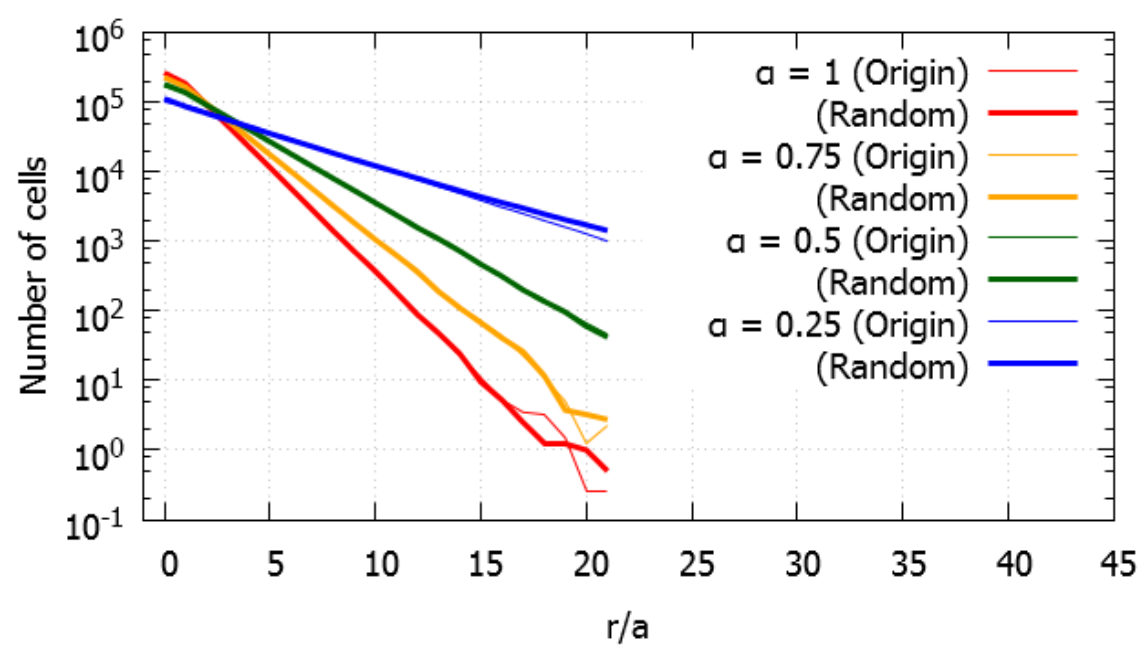

Fig. 2. Spatial distributions of cells. (a) The cells were released from the origin at time $n=0$. Thin and thick lines indicate time steps $n=299$ and $n=300$, respectively. Two almost-converged distributions alternatively appear at every second time step. (b) Thin and thick lines indicate that the cells were released from the origin at time $n=0$ and were randomly distributed at time $n=0$, respectively. The cell numbers in Panel (b) are averaged from the cell numbers at $n=299$ and $n=300$. The decay depends on $\alpha$ but not on the initial distribution. 


\subsection{Simulation results}

The rules in subsection 2.1 were implemented in a simulation of $10^{6}$ cells released at time $n=0$. No intercellular interactions were assumed. Two initial distributions were adopted: (i) all cells were gathered at the origin, (ii) cells were randomly distributed in the region $r / a \leq 30$, where $r$ denotes the distance from the origin.

Figure 2 plots the spatial distributions of the cells at approximately 300 time steps. Under initial condition (i), the cells are absent at even positions $r / a$ at even time, and at odd positions $r / a$ at odd time. As shown in the figure, the converged distributions at even and odd time alternate as time elapses. Therefore, the average of the two distributions is plotted in Fig. 2(b). Both the initial distributions appear to converge to a single distribution. Each distribution is a geometric sequence whose common ratio depends on the parameter $\alpha$.

From Fig. 2(b), the decay in the probability distribution is roughly estimated as

$$
p(r) \propto \frac{1}{(1+\alpha)^{r / a}} .
$$

\subsection{Steady probability distribution sequentially decreases at every interval}

Let us find a steady distribution that satisfies rules (a) and (b) defined in subsection 2.1. Figure 3 indicates the probability propagation along the positive region of the number line. $L$ and $R$ denote that the probability propagates from the left and right sides, respectively. $L_{i}^{n+1}$ depends on the probability at position $i-1$ at time $n$. The probability $L_{i-1}^{n}$ corresponds to the cell's motion from the left side, wherein the cell previously receded from the origin. Therefore, the cell will tumble and $L_{i-1}^{n}$ propagates to $R_{i-2}^{n+1}$ or $L_{i}^{n+1}$ with equal probability $(1 / 2)$. The probability $R_{i-1}^{n}$ corresponds to the cell's motion from the right side, wherein the cell previously approached the origin. The cell will continue toward the origin with probability $\alpha$ and will tumble with probability $1-\alpha$. $R_{i-1}^{n-1}$ propagates to the left or right with probabilities of $\alpha+1 / 2(1-\alpha)=1 / 2 \cdot(1+\alpha)$ or $1 / 2 \cdot(1-\alpha)$, respectively (see Fig. 3). Thus we have

$$
L_{i}^{n+1}=\frac{1}{2} L_{i-1}^{n}+\frac{1}{2}(1-\alpha) R_{i-1}^{n} .
$$

Similarly,

$$
R_{i}^{n+1}=\frac{1}{2} L_{i+1}^{n}+\frac{1}{2}(1+\alpha) R_{i+1}^{n} .
$$

\section{Origin (Source of attractant chemical)}

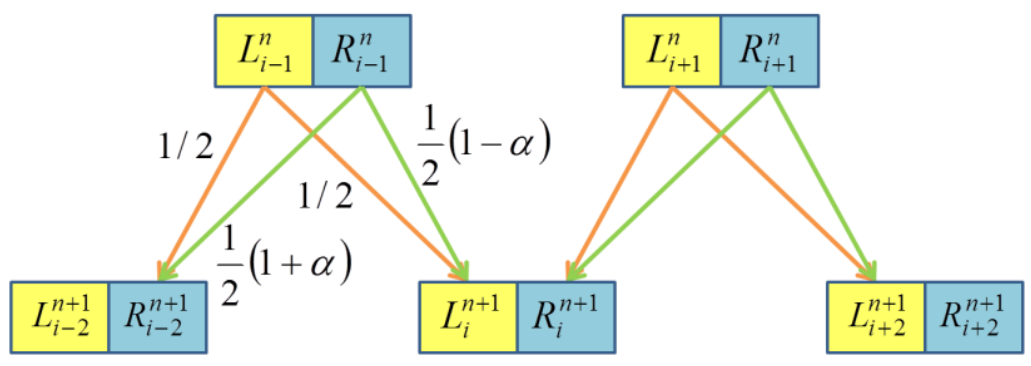

Fig. 3. Time-position probability diagram of cell dynamics along the right half of the number line. The chemoattractant is sourced from the left of this diagram.

$L$ and $R$ denote the probability of propagation from the left and right, respectively. For example, $L_{i}^{n+1}$ means the probability of being at position $i$ at time $n+1$, contributed by $1 / 2 \cdot L_{i-1}^{n}$ and $1 / 2 \cdot(1-\alpha) R_{i-1}^{n}$. 
Eqs. (2) and (3) are the present model. They are recurrence relations (Goldstein, 1951, Kac, 1974) slightly modified to consider the different direction change probabilities between right-moving and left-moving cells (Codling, et al., 2008, Schnitzer, 1993).

Summing these probabilities, we obtain the probability of being at position $i$ at time $n+1$ :

$$
p_{i}^{n+1}=L_{i}^{n+1}+R_{i}^{n+1}=\frac{1}{2}\left(p_{i+1}^{n}+p_{i-1}^{n}\right)+\frac{1}{2} \alpha\left(R_{i+1}^{n}-R_{i-1}^{n}\right) .
$$

Shifting the position $i$ to $i+1$ in Eq. (2) and $i$ to $i-1$ in Eq. (3), and summing them, we have

$$
R_{i+1}^{n}-R_{i-1}^{n}=p_{i+1}^{n}-p_{i}^{n-1} .
$$

Substituting Eq. (5) into Eq. (4), we obtain

$$
p_{i}^{n+1}=\frac{1}{2}(1+\alpha) p_{i+1}^{n}+\frac{1}{2} p_{i-1}^{n}-\frac{\alpha}{2} p_{i}^{n-1} \text {. }
$$

Thus, in a steady state, as $p_{i}^{n+1}=p_{i}^{n}=p_{i}^{n-1}$ the probability is a geometric sequence of the position number. Let $\lambda$ be the common ratio of the geometric sequence, that is, $p_{i+1}=\mu p_{i}$. Then, Eq. (7) yields

$$
(\mu-1)\{(1+\alpha) \mu-1\}=0 .
$$

The former $\mu=1$ corresponds to a uniform distribution, which is inappropriate for adjusting the boundary conditions in the presence of a chemoattractant source. The latter solution gives

$$
p_{i} \propto \frac{1}{(1+\alpha)^{i}} .
$$

This is equivalent to Eq. (1), as suggested by the simulation results.

Shifting the position $i$ to $i-1$ in Eq. (3), and substituting it into Eq. (2), we obtain

$$
p_{i}^{n+1}-R_{i}^{n+1}=\frac{1}{2} p_{i-1}^{n}-\frac{\alpha}{2}\left(\frac{1}{2} p_{i}^{n}+\frac{\alpha}{2} R_{i}^{n}\right),
$$

which yields, in steady state,

$$
R_{i}=\frac{1}{2+\alpha} p_{i}, L_{i}=p_{i}-R_{i}=\frac{1+\alpha}{2+\alpha} p_{i} .
$$

Thus, the ratio of $L$ to $R$ is common at all positions in steady state.

\subsection{Steady probability distribution decreasing sequentially at every two intervals}

In subsection 2.2, we identified four stable distributions. The first and second distributions occur at even and odd positions, respectively, at even time. The third and fourth distributions occur at even and odd positions, respectively, at odd time. We seek a probability distribution at every second position at every second time which is derived from Eq. (6).

Using Eq. (6) recursively and substituting Eq. (5) into Eq. (4), we obtain

$$
\begin{aligned}
p_{i}^{n+1} & =\frac{1}{2}(1+\alpha) p_{i+1}^{n}+\frac{1}{2} p_{i-1}^{n}-\frac{\alpha}{2} p_{i}^{n-1} \\
& =\frac{1}{2}(1+\alpha)\left\{\frac{1}{2}(1+\alpha) p_{i+2}^{n-1}+\frac{1}{2} p_{i}^{n-1}-\frac{\alpha}{2} p_{i+1}^{n-2}\right\}+\frac{1}{2}\left\{\frac{1}{2}(1+\alpha) p_{i}^{n-1}+\frac{1}{2} p_{i-2}^{n-1}-\frac{\alpha}{2} p_{i-1}^{n-2}\right\}-\frac{\alpha}{2} p_{i}^{n-1} .
\end{aligned}
$$

Shifting the time $n+1$ to $n-1$ in Eq. (6), Eq. (6) yields

$$
\frac{1}{2}(1+\alpha) p_{i+1}^{n-2}+\frac{1}{2} p_{i-1}^{n-2}=p_{i}^{n-1}+\frac{\alpha}{2} p_{i}^{n-3} \text {. }
$$

Substituting Eq. (11) into Eq. (10), we obtain

$$
p_{i}^{n+1}=\frac{1}{4}(1+\alpha)^{2} p_{i+2}^{n-1}+\frac{1}{2}(1-\alpha) p_{i}^{n-1}+\frac{1}{4} p_{i-2}^{n-1}-\frac{\alpha^{2}}{4} p_{i}^{n-3} \text {. }
$$

Equation (12) indicates the probability distribution that appears at every second position at every second time. 
In stable state at every second time, $p_{i}^{n+1}=p_{i}^{n-1}=p_{i}^{n-3}$. Let $\sigma$ be the common ratio of the geometric sequence at every second position, that is, $p_{i+2}=\sigma p_{i}$. Then, Eq. (12) gives

$$
\sigma=\frac{1}{(1+\alpha)^{2}}=\mu^{2},
$$

as it is expected from Eq. (7).

If the stable distribution at time even (odd) $m$ is

$$
p_{k+2 j}^{m}=\frac{p_{k}}{(1+\alpha)^{2 j}},
$$

where $p_{k}(k=0,1)$ denotes the constant probability at time $m$ and $j=0$, then the stable distribution during the next odd (even) time is calculated by using Eq. (6) as

$$
p_{k+2 j+1}^{m+1}=\frac{p_{k}}{(1+\alpha)^{2 j+1}} .
$$

Therefore, the first and fourth distributions described at the beginning of this subsection can be combined. Similarly, the second and third distributions can be combined. In this way, we obtain two stable distributions, one appearing at even (odd) positions at even (odd) time; the other appearing at even (odd) positions at odd (even) time with a common ratio of $1 /(1+\alpha)^{2}$.

\section{Approximated continuum model deduced from the discrete model}

Expressing Eq. (6) as a Taylor series, we obtain the continuum model as an approximation of Eq. (6),

$$
\tau \frac{\partial p}{\partial t}=\frac{\alpha}{2-\alpha} a \frac{\partial p}{\partial x}+\frac{2+\alpha}{2-\alpha} \frac{a^{2}}{2} \frac{\partial^{2} p}{\partial x^{2}}+O\left(\tau^{2}\right)+O\left(a^{3}\right)
$$

where $\tau$ is the temporal interval in the discrete model. Note that the $2-\alpha$ in the denominators in the right hand terms are due to the last term at time $n-1$ in Eq. (6). When a typical length scale of the cell distribution is greater than $a$, and a typical time scale is longer than $\tau$, as it is seen in nature, the residual terms will be neglected.

If the diffusion coefficient $D$ is defined as

$$
D=\frac{2+\alpha}{2-\alpha} \frac{a^{2}}{2 \tau},
$$

the advection (or drift) speed is

$$
U=\frac{\alpha}{2-\alpha} \frac{a}{\tau}=\frac{\alpha}{2+\alpha} \frac{2}{a} D
$$

Imposing the conditions

$$
\int_{0}^{\infty} p(x) d x=\frac{1}{2}, p(\infty)=0,
$$

the steady probability distribution satisfying Eq. (16) is

$$
p(x)=\frac{D}{U} e^{-\frac{U}{D} x}=\frac{a}{2} \frac{2+\alpha}{\alpha} e^{-\frac{2 \alpha}{2+\alpha} \frac{x}{a}} .
$$

The cases $\alpha=1$ and $\alpha=0$ correspond to maximally biased and completely random motion of the bacterial cells, respectively. The probability distribution of fully random motions will spread out after sufficiently long time.

We now compare the continuous distribution expressed by Eq. (20) with the discrete distribution expressed by Eq. (7). The reciprocal ratios of the probability distributions between two spatial intervals are presented in Table 1. Both equations yield similar results. 
Table 1. Reciprocals of discrete (2nd row, Eq. (7)) and continuous (3rd row, Eq. (20) or (31)) probability distributions ratios at two positions separated by $a$. The numbers in 4 th row are corresponding values of Eq. (30).

\begin{tabular}{|c|c|c|c|c|}
\hline \hline$\alpha$ & 1 & 0.75 & 0.5 & 0.25 \\
\hline \hline $1+\alpha$ & 2 & 1.75 & 1.5 & 1.25 \\
\hline$e^{\frac{2 \alpha}{2+\alpha}}$ & 1.948 & 1.725 & 1.492 & 1.249 \\
\hline$e^{\frac{\alpha}{2}}$ & 1.649 & 1.455 & 1.284 & 1.133 \\
\hline \hline
\end{tabular}

\section{Discussions}

The present model, represented by Eqs. (2) and (3), is a simple and direct presentation of the features of bacterial chemotaxis. In a correlated random walk, a cell tends to move in the same direction as its previous motion. This is treated as a telegraph equation or a biased telegraph equation (Codling, et al., 2008, Okubo and Levin, 2001, Othmer, et al., 1988, Schnizer, 1993). Eq. (16) derived from the present model, is different from telegraph equations. Moreover, the result of the present model gives a small correction of the steady solution of the telegraph equation.

Let $r(x, t)$ be a probability density of cells moving to the right and $l(x, t)$ be the probability density moving to the left. At each time step $\tau$, each of right-moving cells either changes its direction with a constant rate $\lambda_{r}$ and moves a distance $a$ or moves a distance $a$ in the previously moving direction at a rate $1-\lambda_{r}$. Similarly, each of left moving cell changes its direction at a constant rate $\lambda_{l}$ or continuously moves at a rate $1-\lambda_{l}$. Taking the limit $\tau \rightarrow 0, a \rightarrow 0$ such that $a / \tau=v$, we obtain the equations

$$
\begin{aligned}
& \frac{\partial r}{\partial t}=-v \frac{\partial r}{\partial x}-\lambda_{r} r+\lambda_{l} l, \\
& \frac{\partial l}{\partial t}=v \frac{\partial l}{\partial x}+\lambda_{r} r-\lambda_{l} l .
\end{aligned}
$$

A biased telegraph equation is derived from Eqs. (21a) and (21b) (Codling, et al., 2008). The steady probability distribution $p=r+l$ is

$$
p(x)=p\left(x_{0}\right) \exp \left(-\frac{\lambda_{r}-\lambda_{l}}{v} x\right),
$$

where $x_{0}$ is a reference point (Schnizer 1993).

In the present model, each of cells from the left (right-moving) changes its direction with a probability $\lambda_{r} \tau(=1 / 2)$, and each of cells from the right (left-moving) changes its direction with a probability $\lambda_{l} \tau(=1 / 2 \cdot(1-\alpha))$. Using $\lambda_{r} \tau$ and $\lambda_{l} \tau$, Eqs. (2) and (3) are written

$$
\begin{aligned}
& L_{i}^{n+1}=\left(1-\lambda_{r} \tau\right) L_{i-1}^{n}+\lambda_{l} \tau R_{i-1}^{n}, \\
& R_{i}^{n+1}=\lambda_{r} \tau L_{i+1}^{n}+\left(1-\lambda_{l} \tau\right) R_{i+1}^{n} .
\end{aligned}
$$

The derivation of the steady probability distribution from Eqs. (23a) and (23b) is similar to the derivation of Eq. (7),

$$
p_{i} \propto\left(\frac{1-\lambda_{r} \tau}{1-\lambda_{l} \tau}\right)^{i} .
$$

Expanding Eqs. (23a) and (23b) about time $n$ and position $i$ up to $O(\tau)$ and $O\left(a^{2}\right)$ like Eq. (16) gives 


$$
\begin{aligned}
& \frac{\partial L}{\partial t}=-v \frac{\partial L}{\partial x}-\lambda_{r} L+\lambda_{l} R+\lambda_{r} \frac{\partial L}{\partial x}-\lambda_{l} \frac{\partial R}{\partial x}+\frac{1}{2} v a\left(1-\lambda_{r}\right) \frac{\partial^{2} L}{\partial x^{2}}+\frac{1}{2} v a \lambda_{l} \frac{\partial^{2} R}{\partial x^{2}}, \\
& \frac{\partial R}{\partial t}=v \frac{\partial R}{\partial x}+\lambda_{r} L-\lambda_{l} R+\lambda_{r} \frac{\partial L}{\partial x}-\lambda_{l} \frac{\partial R}{\partial x}+\frac{1}{2} v a \lambda_{r} \frac{\partial^{2} L}{\partial x^{2}}+\frac{1}{2} v a\left(1-\lambda_{l}\right) \frac{\partial^{2} R}{\partial x^{2}}
\end{aligned}
$$

Additional terms appear in Eqs. (25a) and (25b) compared with Eqs. (21a) and (21b). This is because that the direction changes of cells occur at an instance between two consecutive runs taking finite time in the present model. In contrast, in Eqs. (21a) and (21b), the direction changes continually occur during the time period $\tau$. Eqs. (21a) and (21b) can be rewritten in the integrated form showing the conservation of the number of cells in a control volume. In contrast, small leakage of cells due to the truncation of expansion is allowed in Eqs. (25a) and (25b). Summing Eqs. (25a) and (25b), we have

$$
\frac{\partial p}{\partial t}=-v \frac{\partial(L-R)}{\partial x}+2 \lambda_{r} \frac{\partial L}{\partial x}-2 \lambda_{l} \frac{\partial R}{\partial x}+\frac{1}{2} v a \frac{\partial^{2} p}{\partial x^{2}} .
$$

Similarly, shifting the position $i$ to $i+1$ in Eq. (2) and $i$ to $i-1$ in Eq. (3), expanding them, and summing them, we obtain

$$
\frac{\partial p}{\partial t}=-v \frac{\partial(L-R)}{\partial x}-\frac{1}{2} v a \frac{\partial^{2} p}{\partial x^{2}} .
$$

Eliminating $L$ and $R$ from Eqs. (26) and (27), we have a diffusion-advection equation

$$
\frac{\partial p}{\partial t}=v \frac{\lambda_{r}-\lambda_{l}}{\lambda_{r}+\lambda_{l}} \frac{\partial p}{\partial x}+\frac{1}{2} v a \frac{2-\lambda_{r}-\lambda_{l}}{\lambda_{r}+\lambda_{l}} \frac{\partial^{2} p}{\partial x^{2}} .
$$

Therefore, the steady distribution is

$$
p(x)=p\left(x_{0}\right) \exp \left(-\frac{\lambda_{r}-\lambda_{l}}{2-\lambda_{r}-\lambda_{l}} \frac{x}{a}\right) .
$$

If we set $\lambda_{r} \tau=1 / 2$ and $\lambda_{l} \tau=1 / 2 \cdot(1-\alpha)$, Eqs. (22) and (29) yield

$$
p(x)=p\left(x_{0}\right) \exp \left(-\frac{\alpha}{2} \frac{x}{a}\right)
$$

and

$$
p(x)=p\left(x_{0}\right) \exp \left(-\frac{2 \alpha}{2+\alpha} \frac{x}{a}\right),
$$

respectively. In Table 1, the reciprocal ratios of the probability distributions between two spatial intervals in Eq. (30) are also presented. If $\alpha$ is big, the difference may be significant. Since the present model follows the feature that a bacterial cell continues swimming during a certain period, the model modifies the results obtained by Eq. (30).

\section{Conclusions}

Previously, we proposed a one-dimensional discrete biased random walk model to describe the chemotactic behavior of bacteria. In this study, the model was modified to vary the bias intensity, defined as the probability at which a cell continues to move toward a chemoattractant.

The steady distribution of the cells around the chemical source was numerically simulated. The distribution is a geometric progression in terms of distance from the source. The common ratio of the progression depends on the bias intensity.

Analysis of the distribution revealed two steady, alternating distributions: one appearing at even (odd) positions at even (odd) time and the other appearing at odd (even) positions at even (odd) time. Both distributions are geometric progressions with the same common ratio, which depends on the bias intensity.

The corresponding approximated continuum model is a diffusion-advection equation, not a biased telegraph equation derived from similar analyses. The advection speed is a function of the bias intensity. The analytical solution of the continuum model is consistent with the solutions of the discrete model. 
The bias intensity may reveal the relation between the behavior of an individual cell and their collective migration. To verify this idea, we need to collect bacterial cell's data on the time period between two consecutive tumbles and the spatial length of run and compare with experimental data of bacterial cell distributions around a chemoattractant.

\section{Acknowledgment}

This work was supported by JSPS KAKENHI Grant Number 15K05796. The authors acknowledge the anonymous reviewers for their helpful comments.

\section{References}

Adler, J., Chemotaxis in bacteria, Science, Vol. 153 (1966), pp. 708-716.

Berg, H. C., E. coli in motion (2004), Chapter 4, Springer.

Berg, H. C., Random walks in biology (1993), Chapter 1, Princeton University Press.

Codling, E. A., Plank, M. J. and Benhamou, S., Random walk models in biology, Journal of the Royal Society Interface, Vol. 5 (2008), pp. 813-834.

Goldstein, S., On diffusion by discontinuous movements, and on the telegraph equation, Quarterly Journal of Mechanics and Applied Mathematics, Vol. 4, Pt. 2 (1951), pp. 129-156.

Goto, T. and Nakai, T., A one-dimensional discrete model of biased random walk relating to bacterial chemotaxis, Journal of Aero Aqua Biomechanisms, Vol. 4, No. 1 (2015), pp.853-858.

Kac, M., A stochastic model related to the telegraph equation, Rocky Mountain Journal of Mathematics, Vol. 4, No. 3 (1974), pp. 498-509.

Kanehl, P. and Ishikawa, T., Fluid mechanics of swimming bacteria with multiple flagella, Physical Review E, Vol. 89 (2014), 042704.

Macnab, R. M. and Koshland, D. E., Jr., The gradient-sensing mechanism in bacterial chemotaxis, Proceedings of the National Academy of Sciences of the United States of America, Vol. 69, No. 9 (1972), pp. 2509-2512.

Okubo, A. and Levin, S. A., Diffusion and ecological problems: modern perspectives (2001), Chapter 5 Mathematical treatment of biological diffusion by Okubo A. and Grunbaum D., Springer.

Othmer, H. G., Dunbar, S. R. and Alt, W., Models of dispersal in biological systems, Journal of Mathematical Biology, Vol. 26 (1988), pp. 263-298.

Rubik, B. A. and Koshland, D. E., Jr., Potentiation, desensitization, and inversion of response in bacterial sensing of chemical stimuli, Proceedings of the National Academy of Sciences of the United States of America, Vol. 75, No. 6 (1978), pp. 2820-2824.

Schnitzer, M. J., Theory of continuum random walks and application to chemotaxis, Physical Review E, Vol. 48, No. 4 (1993), pp. 2553-2568.

Turner, L., Ryu, W. S. and Berg, H. C., Real-time imaging of fluorescent flagellar filaments, Journal of Bacteriology, Vol. 182, No. 10 (2000), pp. 2793-2801. 